\title{
Recurrent sustained ventricular tachycardia, hypertrophic cardiomyopathy, and apical aneurysm: Electroanatomic map-guided surgical ablation and left ventricular restoration
}

\author{
Kazuya Kobayashi, MD, ${ }^{\mathrm{a}}$ Toshihiro Ohata, MD, $\mathrm{PhD},{ }^{\mathrm{b}}$ Hideki Ueda, $\mathrm{MD}, \mathrm{PhD},{ }^{\mathrm{a}}$ and \\ Kenjiro Miyamoto, MD, ${ }^{\mathrm{b}}$ Sapporo, Japan
}

Hypertrophic cardiomyopathy (HCM) with apical aneurysm is rare but is associated with high incidences of persistent ventricular tachycardia (VT) and sudden cardiac death. Here we describe the successful management of recurrent VT by means of surgical ablation with an electroanatomic mapping system, a recently available modality, and left ventricular (LV) restoration.

\section{CLINICAL SUMMARY}

A 73-year-old woman with a history of midventricular $\mathrm{HCM}$ and LV apical aneurysm received an implantable cardioverter-defibrillator (ICD) for recurrent sustained monomorphic VT. She continued to have frequent recurrence of sustained VT, resulting in refractory cardiogenic shock despite pharmacologic therapy with amiodarone and $\beta$-blockers.

At admission, an electrocardiogram showed sustained monomorphic VT with a cycle length of $380 \mathrm{~ms}$. VT was terminated by electrical cardioversion, and VT frequency decreased with amiodarone. Transthoracic echocardiography demonstrated asymmetric LV hypertrophy with a midventricular obstruction and a $40 \times 47-\mathrm{mm}$ dyskinetic apical aneurysm. Coronary angiography showed no significant stenosis. Electroanatomic endocardial and epicardial voltage mapping of the LV, performed with the EnSite NavX system (St Jude Medical, Inc, St Paul, Minn), revealed an area of low voltage and scar corresponding to the aneurysm (Figure 1, $A$ and $B$ ). A pace map identical to the clinical VT wave was obtained at the rim of apical aneurysm, which was presumed to be the critical slow conduction zone that caused the reentry of VT (Figure 2).

The operation was conducted through a median sternotomy under conventional cardiopulmonary bypass.

\footnotetext{
From the Department of Cardiovascular Surgery, ${ }^{\mathrm{a}}$ KKR Sapporo Medical Center, Sapporo, Japan; and the Division of Cardiology, ${ }^{\mathrm{b}}$ Cardiovascular Center, Teine Keijinkai Hospital, Sapporo, Japan.

Disclosures: Authors have nothing to disclose with regard to commercial support.

Received for publication Dec 13, 2011; revisions received Jan 2, 2012; accepted for publication Jan 16, 2012; available ahead of print Aug 15, 2013.

Address for reprints: Kazuya Kobayashi, MD, Department of Cardiovascular Surgery, KKR Sapporo Medical Center, Hiragishi 1-6, Toyohira-ku, Sapporo, 062-0931,

Japan (E-mail: kakobayashi_tky@me.com).

J Thorac Cardiovasc Surg 2013;146:983-5

$0022-5223 / \$ 36.00$

Copyright (c) 2013 by The American Association for Thoracic Surgery

http://dx.doi.org/10.1016/j.jtcvs.2012.01.090
}
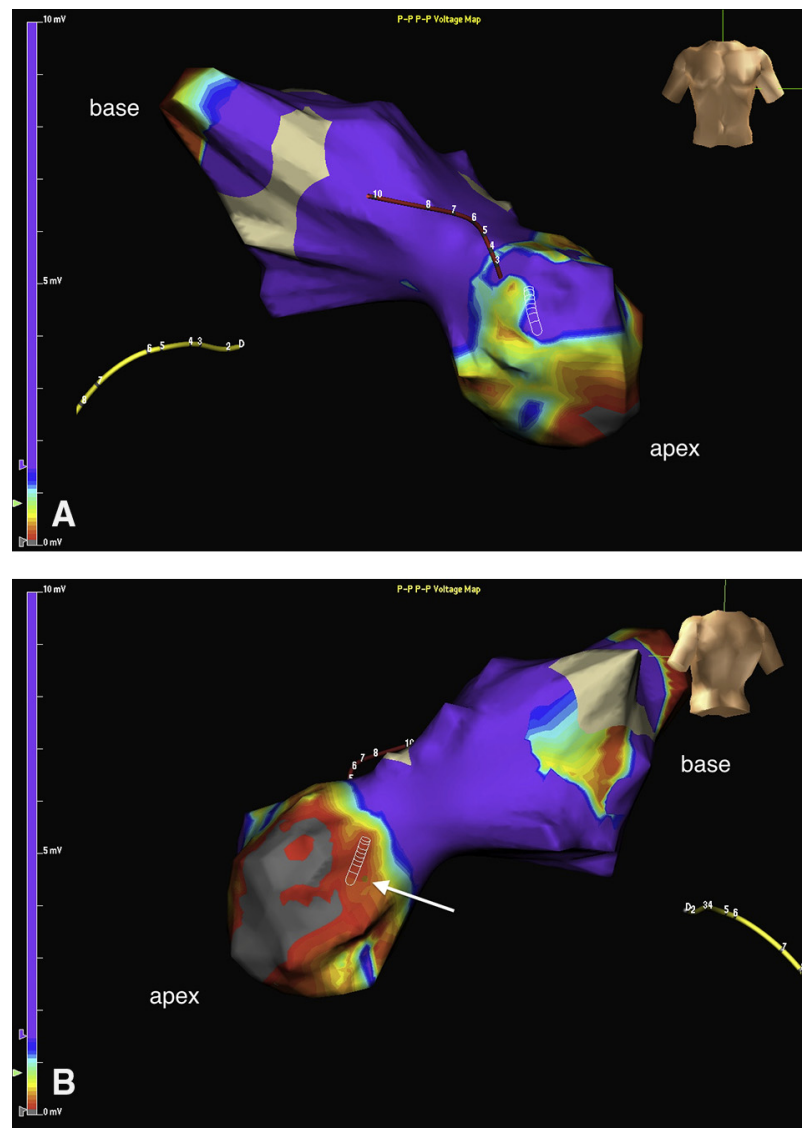

FIGURE 1. Electroanatomic voltage map of the left ventricle during sinus rhythm in anteroposterior (A) and posteroanterior (B) views (EnSite NavX system; St Jude Medical, Inc, St Paul, Minn). Areas with voltage amplitude greater than $1.5 \mathrm{mV}$ are purple. Amplitudes less than $1.5 \mathrm{mV}$ are, in decreasing order, blue, light blue, yellow, orange, red, and gray. The apical chamber is largely a low-voltage area. In the posteroanterior view, the scarred lesion apicolateral wall is gray. Pace mapping detected the critical slow conduction zone that created reentry of ventricular tachycardia at the rim of apical aneurysm, shown as a brown dot (arrow).

Epicardial mapping was done to identify and mark the critical slow conduction zone. Subsequently, radiofrequency ablation was performed with a Cardioblade BP-1 monopolar pen (Medtronic, Inc, Minneapolis, Minn) at the rim of apical aneurysm (the border between normal myocardium and the low-voltage area), including the site identified as critical slow conduction zone, with a target energy of 

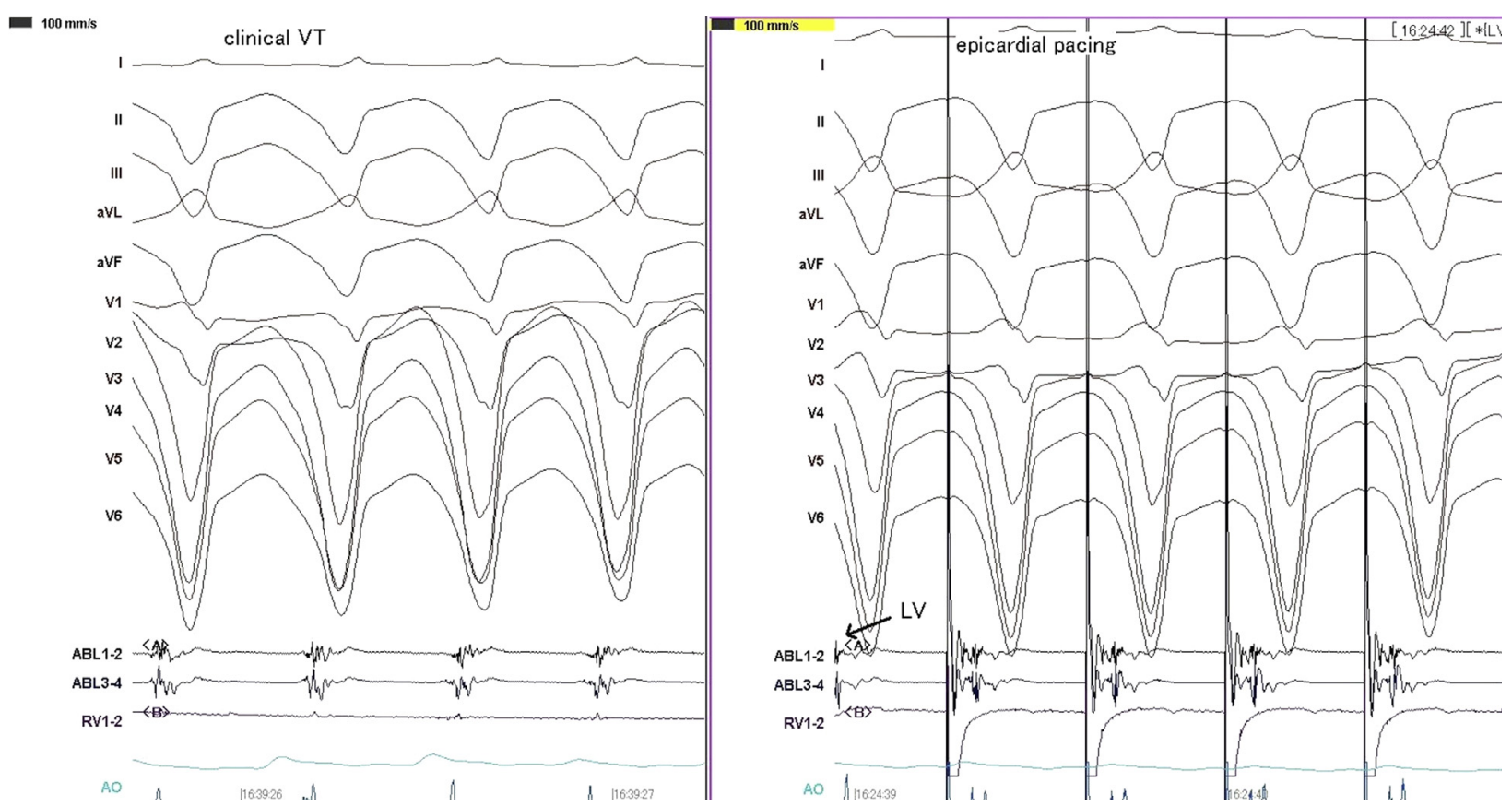

FIGURE 2. Comparison of the 12-lead electrocardiogram during clinical ventricular tachycardia (left) and the pace map during sinus rhythm obtained within the rim of the apical aneurysm (brown dot in Figure 1, B, right).

$21 \mathrm{~W}$ and a maximum impedance of $130 \Omega$ for 60 seconds for each ablation from the epicardial side.

The aneurysm was opened longitudinally with an incision parallel to the left descending artery, and the same zone was cryoablated $\left(-60^{\circ} \mathrm{C}\right.$ for 90 seconds) from both endocardial and epicardial sides. Finally, radiofrequency ablation was added on the same zone from the endocardial side to achieve complete exclusion. Epicardial pacing at the apical lesion revealed no induction of VT and complete electrical isolation of the aneurysm. Excess aneurysmal tissue was excised, and the LV was reconstructed with the endoventricular patch plasty technique advocated by Suma and colleagues.

The postoperative course was uneventful. The patient was discharged and is doing well. At the 1-year followup, the ICD showed no further VT episodes during treatment with amiodarone $(200 \mathrm{mg} / \mathrm{d})$.

\section{DISCUSSION}

HCM associated with LV apical aneurysm is an uncommon subgroup of HCM, but its prognosis is unfavorable. The overall rate of adverse consequences, including sudden death, embolic stroke, and progressive heart failure, is $10.5 \%$ per year-significantly higher than in the general $\mathrm{HCM}$ population. ${ }^{2}$ In addition, HCM with apical aneurysm is associated with a nonsustained VT in more than $40 \%$ of patients. ${ }^{2}$ Sustained VT is relatively rare but may be underestimated because of early degeneration into ventricular fibrillation. In many cases, electrophysiologic studies show the arrhythmogenic substrate for VT to be located at the rim of aneurysm, in the border zone between the scarred, fibrous apical region and the intact myocardium.

Management of sustained monomorphic VT associated with HCM and apical aneurysm is difficult, because the VT associated with apical aneurysm is generally refractory to pharmacologic therapy. Although ICD implantation effectively prevents sudden cardiac death, an ICD alone cannot prevent recurrence of VT. Another therapeutic alternative is thus necessary to prevent VT.

Catheter ablation, an important treatment for this purpose can be successful in the short term; however, it may be difficult to ablate thickened hypertrophic myocardial tissue ${ }^{3}$ and to place an ablation catheter into the narrow neck of the aneurysm. ${ }^{4}$ More importantly, because catheter ablation cannot eliminate aneurysmal apical tissue, it cannot eliminate problems that arise from the apical aneurysm, such as thromboembolism and cardiac dysfunction.

Cardiac surgery for VT has become uncommon since the introduction of the ICD and catheter ablation. Nevertheless, surgical therapy by means of direct surgical ablation and ventricular remodeling is a reasonable alternative to control sustained monomorphic VT, thromboembolism, and cardiac dysfunction in patients with HCM and apical aneurysm. In addition, an electroanatomic mapping system may be useful for more accurate surgical ablation because it can clarify the distribution of arrhythmogenic substrate by imaging. 


\section{References}

1. Suma H, Isomura T, Horii T, Nomura F. Septal anterior ventricular exclusion procedure for idiopathic dilated cardiomyopathy. Ann Thorac Surg. 2006;82: 1344-8.

2. Maron MS, Finley JJ, Bos JM, Hauser TH, Manning WJ, Haas TS, et al. Prevalence, clinical significance, and natural history of left ventricular apical aneurysms in hypertrophic cardiomyopathy. Circulation. 2008;118:1541-9.
3. Inada K, Seiler J, Roberts-Thomson KC, Steven D, Rosman J, John RM, et al Substrate characterization and catheter ablation for monomorphic ventricular tachycardia in patients with apical hypertrophic cardiomyopathy. J Cardiovasc Electrophysiol. 2011;22:41-8.

4. Lim KK, Maron BJ, Knight BP. Successful catheter ablation of hemodynamically unstable monomorphic ventricular tachycardia in a patient with hypertrophic cardiomyopathy and apical aneurysm. J Cardiovasc Electrophysiol. 2009;20:445-7. 